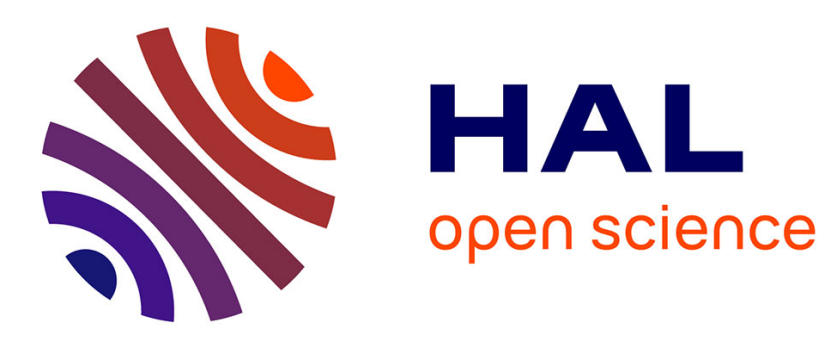

\title{
Heat transfer in the thermo-electro-hydrodynamic convection under microgravity conditions
}

Mireille Tadie Fogaing, Harunori Yoshikawa, Olivier Crumeyrolle, Innocent

Mutabazi

\section{- To cite this version:}

Mireille Tadie Fogaing, Harunori Yoshikawa, Olivier Crumeyrolle, Innocent Mutabazi. Heat transfer in the thermo-electro-hydrodynamic convection under microgravity conditions. European Physical Journal E: Soft matter and biological physics, 2014, 37, pp.35. 10.1140/epje/i2014-14035-0 . hal00987808v2

\section{HAL Id: hal-00987808 \\ https://hal.science/hal-00987808v2}

Submitted on 7 May 2014

HAL is a multi-disciplinary open access archive for the deposit and dissemination of scientific research documents, whether they are published or not. The documents may come from teaching and research institutions in France or abroad, or from public or private research centers.
L'archive ouverte pluridisciplinaire $\mathbf{H A L}$, est destinée au dépôt et à la diffusion de documents scientifiques de niveau recherche, publiés ou non, émanant des établissements d'enseignement et de recherche français ou étrangers, des laboratoires publics ou privés. 


\title{
Heat transfer in the thermo-electro-hdyrodynamic convection under microgravity conditions
}

\author{
M. Tadie Fogaing ${ }^{1}$, H.N. Yoshikawa ${ }^{1 \mathrm{a}}$, O. Crumeyrolle ${ }^{1}$, and I. Mutabazi ${ }^{1 \mathrm{~b}}$ \\ Laboratoire Ondes et Milieux Complexes, UMR 6294 CNRS, Université du Havre \\ 53, rue de Prony, CS80540, 76058 Le Havre Cedex, France \\ Received: date / Revised version: date
}

\begin{abstract}
This article deals with the thermal convection in a dielectric liquid confined in an infinite-length capacitor with a temperature gradient under microgravity conditions. The dielectrophoretic force resulting from differential polarization of the liquid plays the role of buoyancy force with an effective electric gravity. It induces the convection when the Rayleigh number based on this electric gravity exceeds a critical value. Two-dimensional numerical simulation for a geometry with a large aspect ratio is used to determine the developed convective flow. The Nusselt number is computed for different values of Prandtl number and its dependence on the distance from the critical condition is determined. The viscous and thermal dissipations are found to be identical to those in the Rayleigh-Bénard convection which yields the same heat transfer.
\end{abstract}

PACS. 44.24.+f Natural convection - 47.65.-d Magnetohydrodynamics and electrohydrodynamics

\section{Introduction}

Rayleigh-Bénard convection in a fluid layer confined between two horizontal plates with a vertical temperature gradient along the gravitational acceleration $\boldsymbol{g}$ has become the prototype of modern nonlinear physics [1-3]. Similar thermal convections can be induced by using external fields and specific properties of fluids even under microgravity. A magnetic field applied to ferrofluids [4] or an electric field applied to dielectric fluids [5] allow to generate a ponderomotive force which can be regarded as thermal buoyancy in an artificial gravity. In the present work, we are interested in the thermal convection induced by an electric field and a temperature gradient applied to dielectric fluids. The latter convection is often referred to as thermo-electro-hydrodynamic (TEHD) convection. It has been the subject of many investigations since few decades, particularly for the case of dielectric fluids confined between two horizontal parallel plane electrodes [610]. The driving force of the convection arises from the dielectrophoretic (DEP) force density $\boldsymbol{F}_{D E P}=-\frac{1}{2} E^{2} \nabla \epsilon$, where $E$ is the magnitude of the electric field $\boldsymbol{E}$ and $\epsilon$ is the electric permittivity of the fluid. The DEP force is a consequence of differential polarization of the fluid. It is a component of the electrohydrodynamic force $[11,12]$ and dominates over the other components when the applied electric field is alternating with a high frequency $f$ and when the fluid is incompressible and has no mobile

\footnotetext{
a Present address: Laboratoire J.-A. Dieudonné, UMR 7351 CNRS, Université de Nice-Sophia Antipolis

b E-mail: innocent.mutabazi@univ-lehavre.fr
}

boundaries [10]. Under a temperature gradient, the DEP force can be generated through the thermal variation of $\epsilon$ :

$$
\boldsymbol{F}_{D E P}=-\frac{1}{2} E^{2} \nabla \epsilon(T)=-\alpha \rho \theta \boldsymbol{g}_{e}+\nabla\left(\frac{e \theta E^{2}}{2}\right),
$$

where $\theta$ is the deviation from the reference temperature $T_{0}$ : $\theta=T-T_{0}$. The introduced electric gravity $\boldsymbol{g}_{e}$ is proportional to the gradient of the electric energy stored in the fluid:

$$
\boldsymbol{g}_{e}=\frac{e}{\rho \alpha} \nabla\left(\frac{\epsilon_{1} E^{2}}{2}\right) .
$$

In eq. (1), the thermal variation of the permittivity has been modeled by a linear relationship $\epsilon=\epsilon_{1}(1-e \theta)$ with the reference permittivity $\epsilon_{1}$ at $T=T_{0}$. The coefficient $e$ is positive and of the order of $10^{-3}-10^{-2} \mathrm{~K}^{-1}$ for most dielectric fluids. The first term in eq. (1) is the analogue of the Archimedian buoyancy so that a thermal convection will be induced when the Rayleigh number based on the electric gravity $\boldsymbol{g}_{e}$ exceeds a certain critical value. The second term in eq. (1) can be lumped with the hydrodynamic pressure term in the momentum equation.

The DEP force can induce convection even in a stable thermal stratification against the Earth's gravity $\boldsymbol{g}$, i.e., the configuration where the temperature gradient is directed opposite to $\boldsymbol{g}$, as shown in the linear stability theories [6-8]. The nonlinear behavior of the convection just above the critical state has been discussed by Stiles [8] and Yoshikawa et al. [10] by using a weakly nonlinear analysis and DNS, respectively.

Yoshikawa et al. [10] highlighted the role of the perturbation component of the electric gravity. In the unper- 
turbed state (fig. 1a), the thermoelectric buoyancy exerted on a fluid parcel is completely balanced by the pressure. A small perturbation $\delta \theta$ of the temperature of the parcel results in the buoyancy $-\alpha \rho \delta \theta \overline{\boldsymbol{g}}_{e} \mathcal{V}$, where $\overline{\boldsymbol{g}}_{e}$ is the electric gravity in the unperturbed state and $\mathcal{V}$ is the volume of the parcel. It can be directed to the lower temperature zone to destabilize the base state. The temperature perturbation also results in a perturbation electric field $\delta E$ through the thermal variation of $\epsilon$, which is given by $\delta E \sim e \delta \theta \bar{E}$, according to Gauss's law of electricity. The gradient of the electric energy is varied due to $\delta E$ by $-\epsilon_{1} \bar{E} \delta E / D$ and $\epsilon_{1} \bar{E} \delta E / D$ at the upper and lower part of the parcel, where $D$ is the diameter of the parcel: $\mathcal{V}=\pi D^{3} / 6$. The thermoelectric buoyancy due to $\delta E$ can then be estimated as $-e\left(\bar{\theta}+\partial_{z} \bar{\theta} D / 2\right)\left(-\epsilon_{1} \bar{E} \delta E / D\right) \mathcal{V} / 2$ for the upper half of the parcel and as $-e\left(\bar{\theta}-\partial_{z} \bar{\theta} D / 2\right)\left(\epsilon_{1} \bar{E} \delta E / D\right) \mathcal{V} / 2$ for the lower half of the parcel. In the total, the force due to the electric field perturbation is

$$
e \epsilon_{1} \bar{E} \delta E \frac{\partial \bar{\theta}}{\partial z} \frac{\mathcal{V}}{2}=e^{2} \epsilon_{1} \bar{E}^{2} \delta \theta \frac{\partial \bar{\theta}}{\partial z} \frac{\mathcal{V}}{2}
$$

The parcel is, in other words, subjected to a gravity $g_{e}^{\prime}=$ $-e^{2} \epsilon_{1}\left(\partial_{z} \bar{\theta}\right) \bar{E}^{2} / \alpha \rho$. This perturbation gravity is directed opposite to the temperature gradient so that it has a stabilizing effect. This was confirmed in the linear stability theory and the analysis of the DNS results $[10,13]$.

The present study is the continuation of the latter work and aims to develop, using DNS, the nonlinear properties of the TEHD convection in a two dimensional capacitor with an alternating electric tension and a temperature gradient. The paper is organized as follows: in the next section, we present the flow equations and the numerical solution procedure. The results are given and discussed in sect. 3. The last section is concerned with the conclusion.

\section{Flow equations}

We consider a dielectric fluid confined in a capacitor with a temperature gradient and an alternating voltage $V(t)=$ $\sqrt{2} V_{0} \sin (2 \pi f t)$ imposed over the gap between the electrodes. (fig. 2). Characteristic scales can be used to introduce non-dimensional control parameters: the viscous relaxation time $\tau_{\nu}$ for the time, the gap $d$ between the electrodes for the length and $\Delta \theta$ for the temperature. The

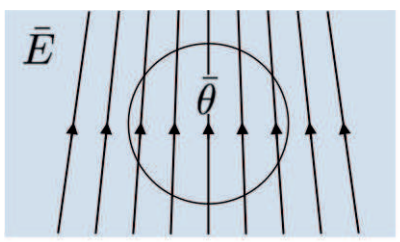

(a) Without perturbation

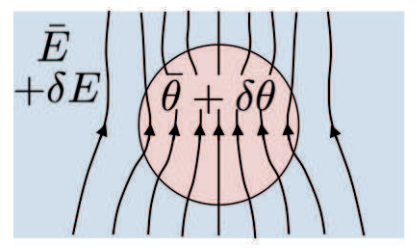

(b) With perturbation
Fig. 1. Electric field perturbation induced by a small temperature perturbation $\delta \theta$ of a parcel of dielectric fluid.

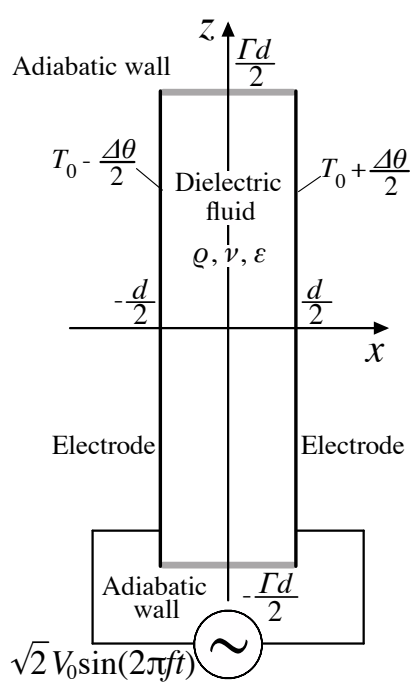

Fig. 2. Geometrical configuration of the problem

velocity is scaled by the diffusion velocity $\nu / d$. The resulting control parameters are the Prandtl number $\operatorname{Pr}=\nu / \kappa$, the electric Rayleigh number $L=\alpha \Delta \theta g_{e, 0} d^{3} / \kappa \nu$, the thermoelectric parameter $B=e \Delta \theta$. The electric gravity at the middle of the gap, $g_{e, 0}$, has been chosen as characteristic electric gravity. It is given by $g_{e, 0}=e \epsilon_{1} V_{0}^{2} B^{3} / \rho \alpha d^{3}[\log \{(1-$ $B / 2) /(1+B / 2)\}]^{2}$. Table 1 shows some values of $g_{e, 0}$ evaluated for different dielectric liquids.

In the electrohydrodynamic Boussinesq approximation, the equations for TEHD convection read:

$$
\begin{aligned}
& \boldsymbol{\nabla} \cdot \boldsymbol{u}=0, \\
& \frac{\partial \boldsymbol{u}}{\partial t}+\boldsymbol{u} \cdot \boldsymbol{\nabla} \boldsymbol{u}=-\nabla H+\nabla^{2} \boldsymbol{u}-\frac{L}{P r} \theta \boldsymbol{g}_{e}, \\
& \frac{\partial \theta}{\partial t}+\boldsymbol{u} \cdot \boldsymbol{\nabla} \theta=\frac{1}{P r} \nabla^{2} \theta, \\
& \boldsymbol{\nabla} \cdot[(1-B \theta) \nabla \phi]=0 .
\end{aligned}
$$

where $H$ is the dimensionless Bernoulli function. In the heat conduction equation (6), the viscous dissipation and Joule heating have been neglected, following the arguments developed by Yavorskaya et al. [14]. These equations must be solved together with boundary conditions at the electrodes $(x= \pm 1 / 2)$ and at the adiabatic walls $(z= \pm \Gamma / 2)$ :

$$
\begin{aligned}
& \boldsymbol{u}=0, \quad \theta=-\frac{1}{2}, \quad \phi=1 \quad \text { at } x=-\frac{1}{2}, \\
& \boldsymbol{u}=0, \quad \theta=\frac{1}{2}, \quad \phi=0 \quad \text { at } x=\frac{1}{2}, \\
& \boldsymbol{u}=0, \quad \partial_{z} \theta=0, \quad \partial_{z} \phi=0 \quad \text { at } z= \pm \frac{\Gamma}{2} .
\end{aligned}
$$

When the electric Rayleigh number $L$ is smaller than a critical value, the homogeneous stationary state $(\boldsymbol{u}, \theta, \phi)=$ $(\mathbf{0}, \bar{\theta}(x), \bar{\phi}(x))$ is established, where

$$
\bar{\theta}=-x, \quad \bar{\phi}=\log \left(\frac{1+B x}{1+B / 2}\right) / \log \left(\frac{1-B / 2}{1+B / 2}\right) .
$$


Table 1. Electric gravity in some dielectric liquids in a capacitor with $d=10 \mathrm{~mm}$ under an electric voltage $V_{0}=10 \mathrm{kV}$ and a temperature difference $1 \mathrm{~K}$. The working temperature is $25^{\circ} \mathrm{C}$.

\begin{tabular}{cccccc}
\hline Liquid & $\rho\left(10^{3} \mathrm{~kg} / \mathrm{m}^{3}\right)$ & $\alpha\left(10^{-3} \mathrm{~K}^{-1}\right)$ & Dielectric constant & $e\left(\mathrm{~K}^{-1}\right)$ & $g_{e, 0}\left(\mathrm{~m}^{2} / \mathrm{s}\right)$ \\
\hline Acetonitrile & 0.777 & 1.38 & 36 & 0.155 & 14.7 \\
Nitrobenzene & 1.198 & 0.830 & 34.9 & 0.188 & 32.2 \\
Acetone & 0.785 & 1.43 & 19.1 & 0.086 & 0.756 \\
Chlorobenzene & 1.101 & 0.985 & 5.61 & 0.0157 & 0.0003 \\
\hline
\end{tabular}
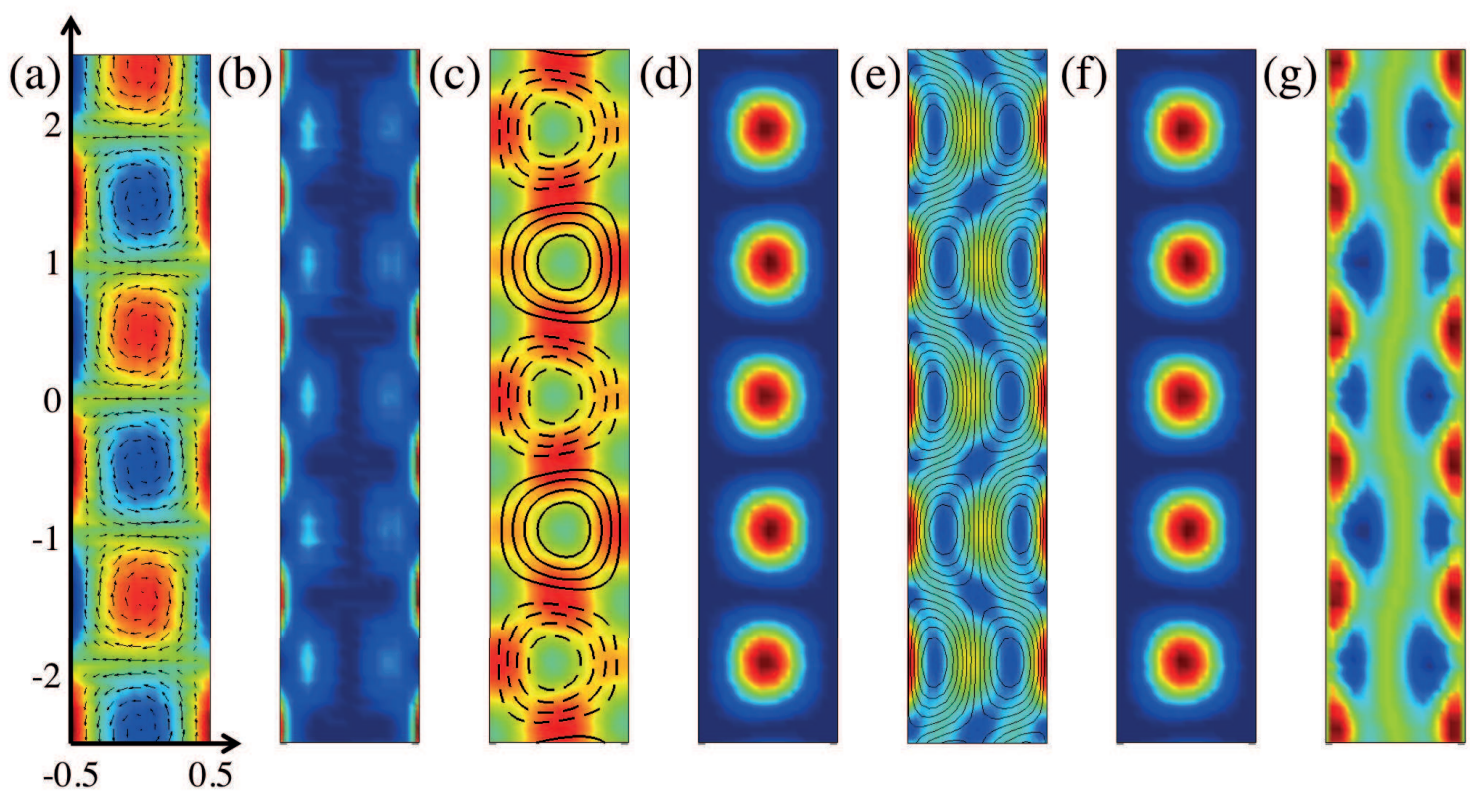

Fig. 3. Saturated flow fields and related quantities at the central part of electrodes: (a) velocity vectors $\boldsymbol{u}^{\prime}$ with vorticity intensity $\left|\nabla \times \boldsymbol{u}^{\prime}\right|$, (b) viscous dissipation function $\Phi$, (c) isotherms $\theta^{\prime}=$ cst (solid and broken lines indicate positive and negative $\theta^{\prime}$, respectively) with thermal dissipation $|\nabla \theta|^{2}$, (d) convective heat transfer $\operatorname{Pr}^{-1} L\left\langle u^{\prime} \theta^{\prime}\right\rangle$, (e) equipotentials $\phi^{\prime}=c s t$ with electric field intensity $\left|\boldsymbol{E}^{\prime}\right|$, (f) power input by the basic electric gravity $w_{B G}$, (g) power input by the perturbation electric gravity $w_{P G}$ and . The basic electric gravity is directed from right to left.

The electric gravity in this base state, $\overline{\boldsymbol{g}}_{e}$, is given by

$$
\overline{\boldsymbol{g}}_{e}=\bar{g}_{e} \boldsymbol{e}_{x} \quad \text { with } \bar{g}_{e}=-\frac{1}{(1+B x)^{3}} .
$$

In the present work, we consider a two-dimensional system with a large aspect ratio $\Gamma=114$ for different values of $\mathrm{Pr}$ in order to simulate the nonlinear behavior of the TEHD convection in an infinite-length capacitor. The thermoelectric parameter will be set at a small value $(B=$ $0.03)$. The set of partial differential equations (4)-(7) with the boundary conditions (8)-(10) are solved by the finite element method implemented in a commercial software package (COMSOL Multiphysics 3.5, Comsol AB, Stockholm, Sweden). Numerical grids are made of identical rectangles with sides of $\Delta x=0.1$ and $\Delta z=0.15$ so that the fluid domain is divided by 10 and 760 along the $x$ and $z$ directions, respectively. The backward differentiation formula is used for the time integration. The convergence of computation was verified by grid refinements. The initial fields are specified as null for the velocities, the temper- ature, and the electric field: The solved problem corresponds to a situation where the electric potential $V_{0}$ and the temperature difference $\theta$ are imposed instantaneously on a steady isothermal fluid layer at $t=0$.

\section{Results}

Velocity, temperature and electric fields have been computed for a given set of parameter values $(\operatorname{Pr}, B, L)$. All the obtained data exhibit a supercritical bifurcation from the conductive state to a convective regime with a critical electric Rayleigh number $L_{c}=2130$. As Yoshikawa et al. [10] have shown, the value of $L_{c}$ is independent from $\operatorname{Pr}$ and coincides with its value determined by the linear stability theory. The critical modes are stationary and independent on $\mathrm{Pr}$.

Hot and cold fluids are, respectively, convected to the cold and hot plates by the thermal buoyancy due to the electric gravity and form convection rolls (fig. 3 a,c). Hot and cold regions are not symmetric with respect to the 


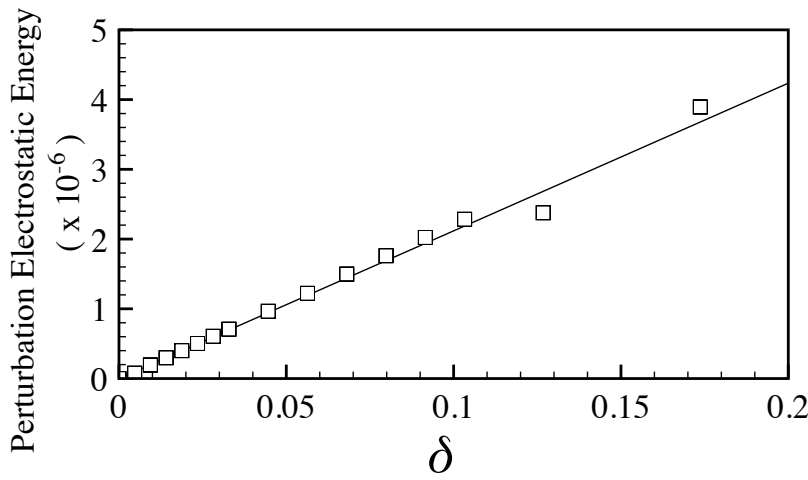

Fig. 4. Perturbation in electrostatic energy $\Delta U_{E}=\left(\epsilon E^{2}-\right.$ $\left.\bar{\epsilon} \bar{E}^{2}\right) / 2(\operatorname{Pr}=100, B=0.03)$.

center line $(x=0)$ and yield a net enhancement of heat transfer by the convection, similar to the Rayleigh-Bénard convection. Perturbative electric fields are concentrated on the electrodes, where the store of the electrostatic energy can be intensified (fig. 3 e). This intensification is actually found but only by a tiny quantity (fig. 4). This means that the convection does not require much energy supply to be stored in the fluid.

In fig. 3, different quantities computed from the fields are also shown $(b, d, f, g)$. To gain a better insight into the TEHD convection, we have used the equations of kinetic energy and temperature variance averaged over the flow domain:

$$
\begin{aligned}
& \frac{d}{d t}\left\langle\frac{1}{2} \boldsymbol{u}^{\prime 2}\right\rangle=\left\langle w_{B G}\right\rangle+\left\langle w_{P G}\right\rangle-\langle\Phi\rangle, \\
& \frac{d}{d t}\left\langle\frac{\theta^{\prime 2}}{2}\right\rangle=\left\langle u^{\prime} \theta^{\prime}\right\rangle-\frac{1}{\operatorname{Pr}}\left\langle\left|\nabla \theta^{\prime}\right|^{2}\right\rangle,
\end{aligned}
$$

where \langle\rangle means the average over the whole fluid domain: \langle\rangle$=\Gamma^{-1} \iint d x d z$, and the primes indicate perturbation components. Different terms $w_{B G}, w_{P G}$ and $\Phi$ in the energy equation (13) are the power input by the basic electric gravity, the power input by the perturbation electric gravity and the viscous dissipation function, respectively:

$$
\begin{aligned}
& w_{B G}=-\frac{L}{P r} \theta^{\prime} \boldsymbol{u}^{\prime} \cdot \overline{\boldsymbol{g}}_{e}, \quad w_{P G}=-\frac{L}{P r}\left(\bar{\theta}+\theta^{\prime}\right) \boldsymbol{u}^{\prime} \cdot \boldsymbol{g}_{e}^{\prime} \\
& \Phi=2\left(\frac{\partial u^{\prime}}{\partial x}\right)^{2}+\left(\frac{\partial u^{\prime}}{\partial z}+\frac{\partial w^{\prime}}{\partial x}\right)^{2}+2\left(\frac{\partial w^{\prime}}{\partial z}\right)^{2}
\end{aligned}
$$

The power input by the basic electric gravity is concentrated at hot and cold cores (fig. $3 \mathrm{f}$ ). The power input by the perturbation electric gravity is positive near the electrodes, while it takes negative values over large zones inside the gap (fig. $3 \mathrm{~g}$ ). The viscous dissipation is primarily due to the shear at the electrodes (fig. $3 \mathrm{~b}$ ).

In the average over the whole fluid domain, the basic electric gravity provide energy to the flow, while the perturbation electric gravity impedes the convection. Figure 5 shows different terms in eq. (13) as a function of the normalized distance $\delta$ from the critical electric Rayleigh

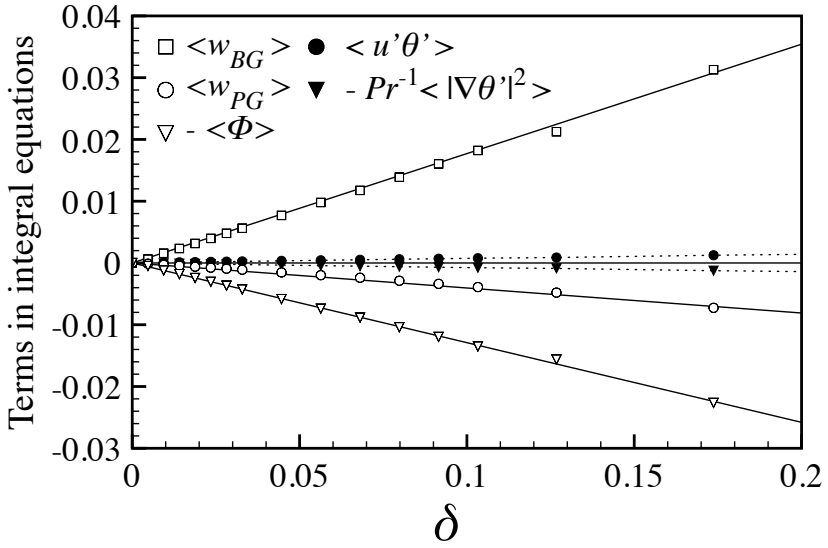

Fig. 5. Different energy generation terms computed after the saturation $(\operatorname{Pr}=100, B=0.03)$.

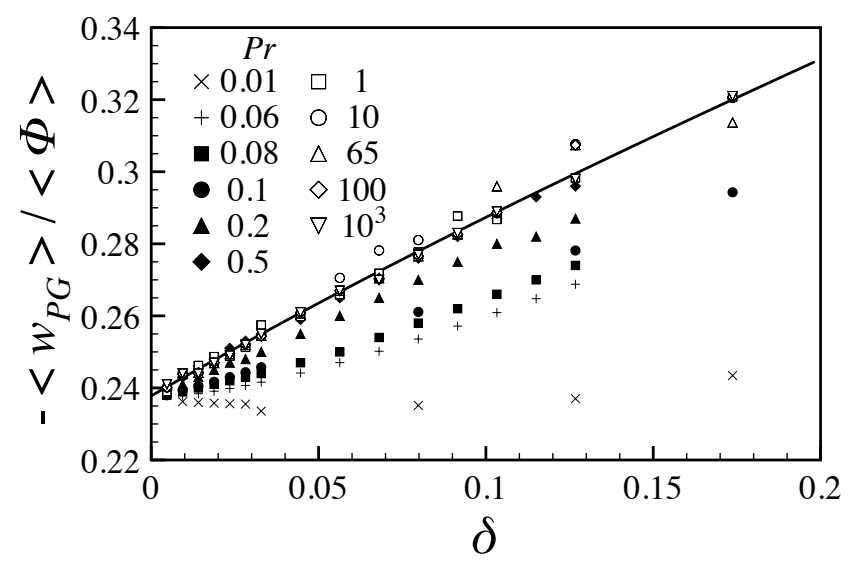

Fig. 6. Relative importance of $W_{P G}$ to $D_{v}$ computed for saturated convection $(B=0.03)$.

number: $\delta=L / L_{c}-1$. The viscous dissipation balances with the contributions from the different electric gravity components. The relative importance of the three contributions is independent of the Prandtl number except for small $\operatorname{Pr}(<1)$. Furthermore, it converges to the same value as $\delta \rightarrow 0$ for all the examined values of $\operatorname{Pr}$ (fig. 6):

$$
\left\langle w_{P G}\right\rangle /\langle\Phi\rangle=-0.24+O(\delta) .
$$

The ratio of $\left\langle w_{B G}\right\rangle$ to $\langle\Phi\rangle$ is given by $\left\langle w_{B G}\right\rangle /\langle\Phi\rangle=$ $-\left\langle w_{P G}\right\rangle /\langle\Phi\rangle+1=1.24+O(\delta)$.

\section{Discussions}

The heat transfer enhancement is given by the Nusselt number $N u$ :

$$
N u=1+\frac{1}{\Gamma} \int_{-\Gamma / 2}^{\Gamma / 2}\left(-\partial_{x} \theta^{\prime}+P r u^{\prime} \theta^{\prime}\right) d z
$$

which compares the convective to conductive heat transfers. 
Yoshikawa et al. [10] showed that the Nusselt number in saturated TEHD convection is proportional to the distance as $N u-1=C \delta$ with the proportionality constant $C=0.78$ for $\operatorname{Pr}$ larger than 1. For small $\operatorname{Pr}$, the constant $C$ varies with $P r$ as it does in the Rayleigh-Bénard convection (fig. 7). Seeking a similar correlation to that for the Rayleigh-Bénard convection [15], one can find

$$
N u-1=\left(1.28-\frac{0.0273}{P r}+\frac{0.0077}{P r^{2}}\right)^{-1} \delta .
$$

For small Prandtl number, the difference in $N u$ between these two convections is small.

In the saturated steady state, one can show that $N u$ can be computed by

$$
N u-1=\operatorname{Pr}\left\langle u^{\prime} \theta^{\prime}\right\rangle .
$$

Making use of eq. (14), one finds that the Nusselt number is given by the averaged value of the thermal dissipation: $N u-1=\left\langle\left|\boldsymbol{\nabla} \theta^{\prime}\right|^{2}\right\rangle$. The latter relationship is also found in the Rayleigh-Bénard convection. For both convections yielding the same heat transfer, the thermal dissipations is identical to each other.

In the limit of small $B$, the basic electric gravity is uniform: $\overline{\boldsymbol{g}}_{e}=-\boldsymbol{e}_{x}$, so that $w_{B G}=\operatorname{Pr}^{-1} L \theta^{\prime} u^{\prime}$. Indeed, $w_{B G}$ and $u^{\prime} \theta^{\prime}$ are identical in our simulation for $B=0.03$ except the factor $\operatorname{Pr}^{-1} L$ (fig. 3 d,f). The heat transfer can then also be computed by

$$
N u-1=\frac{P r}{L}\left\langle w_{B G}\right\rangle=\frac{P r}{L}\left(-\left\langle w_{P G}\right\rangle+\langle\Phi\rangle\right) .
$$

Since the ratio of $\left\langle w_{P G}\right\rangle$ to $\langle\Phi\rangle$ is given by eq. (17) in the vicinity of the criticality, we have $N u-1=1.24 \operatorname{Pr}_{c}^{-1}\langle\Phi\rangle=$ $\operatorname{Pr}\langle\Phi\rangle / 1720$. One can also derive the similar relationship to eq. (21) for the Rayleigh-Bénard convection: $N u-1=$ $\operatorname{Pr} R^{-1}\langle\Phi\rangle[16,17]$. It reads in the vicinity of the criticality $N u-1=\operatorname{Pr}\langle\Phi\rangle / 1708$. For both convections yielding the same heat transfer, the viscous dissipations is hence also be identical to each other.

\section{Conclusion}

The thermo-electro-hydrodynamic convection in dielectric fluids represents a simple way of realizing thermal convection under microgravity conditions. We have highlighted the effect of the perturbative gravity against the destabilization effect of the basic electric gravity. The heat transfer by the TEHD convection has been quantified for different values of the Prandtl number. This effect becomes important for large values of the thermoelectric coupling parameter.

This work benefited from a financial support from CNES (French Space Agency), the CPER-Haute Normandie under the program THETE and from the FEDER. M.T.F. thanks the MESR for financial support during her Ph.D preparation. H.N.Y. acknowledges the financial support of the French National Research Agency (ANR), through the program Investissements d'Avenir (ANR-10 LABX-09-01), LABEX EMC 3 .

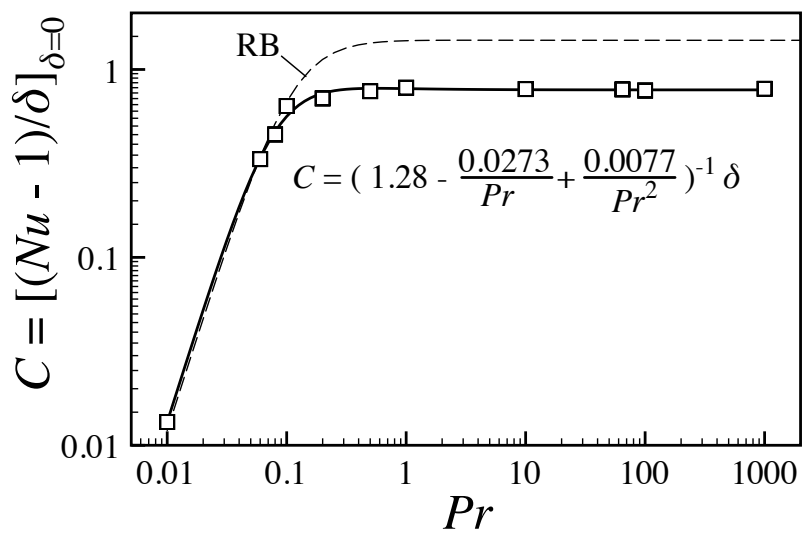

Fig. 7. Proportionality constant $C: N u-1=C \delta$, for different Prandtl numbers $\operatorname{Pr}$ in the vicinity of the criticality $(B=$ 0.03). The broken-line curve is the corresponding constant in the Rayleigh-Bénard convection: $C=(0.6994-0.00472 / \mathrm{Pr}+$ $\left.0.00832 / \operatorname{Pr}^{2}\right)^{-1}\left(R / R_{c}-1\right)[15]$.

\section{References}

1. P. Manneville, Structures dissipatives chaos et turbulence (CEA, Gif-sur-Yvette, 1991)

2. P. Bergé, Y. Pomeau, C. Vidal, Order within chaos: Towards a deterministic approach to turbulence (John Wiley \& Sonc Inc., 1986)

3. M.C. Cross, P.C. Hohenberg, Rev. Mod. Phys. 65(3), 851 (1993)

4. R. Tagg, P.D. Weidman, Z. Angew. Math. Phys. 58, 431 (2007)

5. B. Chandra, D.E. Smylie, Geophysical Fluid Dynamics 3, 211 (1972)

6. P.H. Roberts, Q. J. Mech. Appl. Math. 22, 211 (1969)

7. R.J. Turnbull, Phys. Fluids 12(9), 1809 (1969)

8. P.J. Stiles, Chem. Phys. Lett. 179(3), 311 (1991)

9. B.L. Smorodin, Tech. Phys. Lett. 27(12), 1062 (2001)

10. H.N. Yoshikawa, M.T. Fogaing, O. Crumeyrolle, I. Mutabazi, Phys. Rev. E 87, 043003 (2013)

11. L.D. Landau, E.M. Lifshitz, Electrodynamics of Continuous Media, Vol. 8 of Landau and Lifshitz Course of Theoretical Physics, 2nd edn. (Elsevier ButterworthHeinemann, Burlington, Massachusetts, 1984)

12. J.R. Melcher, Continuum Electromechanics (The MIT Press, 1981)

13. H.N. Yoshikawa, O. Crumeyrolle, I. Mutabazi, Phys. Fluids p. 024106 (2013)

14. I.M. Yavorskaya, N.I. Fomina, Y.N. Belyaev, Acta Astronaut. 11(3-4), 179 (1984)

15. A. Schlüter, D. Lortz, F. Busse, J. Fluid Mech. 23, 129 (1965)

16. B.I. Shraiman, E.D. Siggia, Phys. Rev. A 42(6), 3650 (1990)

17. E.D. Siggia, Annu. Rev. Fluid Mech. 26, 137 (1994) 